\title{
Papers
}

\section{Cost effectiveness of ward based non-invasive ventilation for acute exacerbations of chronic obstructive pulmonary disease: economic analysis of randomised controlled trial}

\author{
P K Plant, J L Owen, S Parrott, M W Elliott
}

\begin{abstract}
Objective To evaluate the cost effectiveness of standard treatment with and without the addition of ward based non-invasive ventilation in patients admitted to hospital with an acute exacerbation of chronic obstructive pulmonary disease.

Design Incremental cost effectiveness analysis of a randomised controlled trial.

Setting Medical wards in 14 hospitals in the United Kingdom.

Participants The trial comprised 236 patients admitted to hospital with an acute exacerbation of chronic obstructive pulmonary disease and mild to moderate acidosis ( $\mathrm{pH} 7.25-7.35)$ secondary to respiratory failure. The economic analysis compared the costs of treatment that these patients received after randomisation.

Main outcome measure Incremental cost per in-hospital death.

Results 24/118 died in the group receiving standard treatment and 12/118 in the group receiving non-invasive ventilation $(\mathrm{P}=0.05)$. Allocation to the group receiving non-invasive ventilation was associated with a reduction in costs of $£ 49362$ (\$78 741; €73 109), mainly through reduced use of intensive care units. The incremental cost effectiveness ratio was - $£ 645$ per death avoided (95\% confidence interval - £2310 to £386), indicating a dominant (more effective and less costly) strategy. Modelling of these data indicates that a typical UK hospital providing a non-invasive ventilation service will avoid six deaths and three to nine admissions to intensive care units per year, with an associated cost reduction of $£ 12000-53000$ per year.

Conclusions Non-invasive ventilation is a highly cost effective treatment that both reduced total costs and improved mortality in hospital.
\end{abstract}

\section{Introduction}

Non-invasive ventilation in the intensive care unit has been shown to reduce the need for intubation and the in-hospital mortality associated with severe exacerbations of chronic obstructive pulmonary disease. ${ }^{1-4}$ It is also feasible and effective in the ward environment for patients who are less severely ill. In a randomised con- trolled trial with 14 participating centres we have shown that non-invasive ventilation reduces the need for intubation by $44 \%$ and in-hospital mortality by $50 \%{ }^{5}$ These results were, however, obtained at a price that included the training of staff, the provision of equipment, and the consumption of additional nursing time. ${ }^{6}$ For non-invasive ventilation to be implemented widely it is necessary to show that the technique is cost effective in the context of the trial and also to model costs in a real life scenario. We report a health economic analysis of the randomised controlled trial, which models the costs and effects of providing a noninvasive ventilation service in a typical hospital in the United Kingdom for patients with chronic obstructive pulmonary disease and mild to moderate respiratory acidosis.

\section{Subjects and methods}

The economic analysis was an incremental cost effectiveness analysis, ${ }^{7}$ performed alongside a 14 centre randomised controlled trial (figure), which compared standard medical treatment (see box) with standard treatment plus non-invasive ventilation (see box) in patients admitted to hospital with an exacerbation of chronic obstructive pulmonary disease, respiratory acidosis $(\mathrm{pH} 7.25-7.35)$, and a respiratory rate greater than 23 breaths per minute. ${ }^{5}$ The nurses administered non-invasive ventilation according to a predefined protocol until the morning of a patient's fourth day. The principal clinical outcomes were the need for intubation, using predefined criteria ( $\mathrm{pH}<7.20$; $\mathrm{pH} 7.20-7.25$ on two occasions 1 hour apart; hypercapnic coma; $\mathrm{PaO}_{2}<6 \mathrm{kPa}$ despite maximum tolerated $\mathrm{FiO}_{2}$; cardiorespiratory arrest) and in-hospital mortality. ${ }^{5}$ Once a patient met the criterion "need for intubation," the attending doctor was free to offer ventilatory support (invasively or non-invasively) at his or her discretion. The primary outcome measure for the economic analysis was mortality in hospital. We conducted the economic evaluation from the perspective of the NHS and hence included only direct costs to the hospitals providing acute care.

\section{Costs}

We identified and valued three categories of costs-for wards, non-invasive ventilation, and intensive care
Department of Respiratory Medicine, St James's University Hospital, Leeds LS9 7TF

P K Plant consultant physician in respiratory medicine

J L Owen respiratory nurse specialist

M W Elliott consultant in respiratory medicine Centre for Health Economics, University of York, York YO10 5DD

S Parrott

lecturer in halth economics

Correspondence to: P K Plant paul.plant@ leedsth.nhs.uk

bmj.com 2003;326:956 


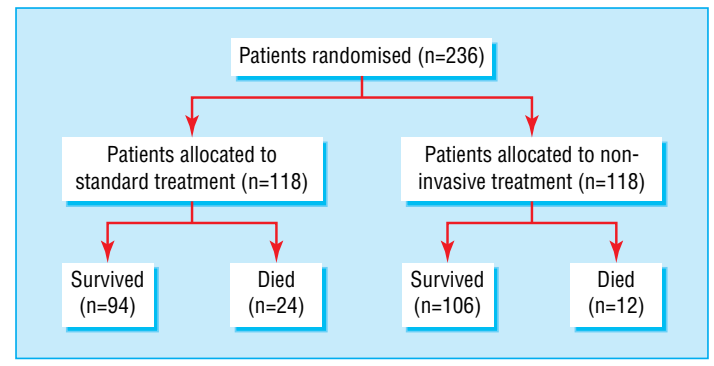

Profile of randomised controlled trial of 236 patients in 14 centres in the United Kingdom

units. We estimated the valuations used for the financial year 1997-8 and derived them from the units participating in the study, using a bottom up approach.

Ward costs-Ward costs consisted of costs for nursing staff, pharmacy, and overheads such as heating, lighting, and costs of buildings. Nursing staff provided non-invasive ventilation for the first three days of the admission. We identified additional nursing time that was attributable to non-invasive ventilation by using a log kept at the end of the bed for the first five days of the admission, on which duration of activity and the seniority (grade) of the nurse were recorded. We calculated the cost of nursing by using the cost of a bed day on each ward, multiplied by the length of stay, and by adding the extra cost of nursing that we identified from the log. We derived pharmacy costs from the standard treatment protocol and allocated these in relation to length of stay and valued from the British National Formulary (March 1997). Each finance department provided daily overhead costs and allocated these in relation to length of stay. In this incremental analysis we assumed costs for investigations and wards to be equal in the two study groups.

Costs of non-invasive ventilation-The costs of non-invasive ventilation included the cost of the initial purchase of the ventilator and selection of masks, replacement of consumables, annual servicing, and training of staff. We treated the initial purchase as a capital purchase with three year and two year life spans for the ventilators and masks, respectively. We applied a dis-

\section{Interventions}

Standard medical treatment (118 patients) Controlled oxygen to maintain $\mathrm{Spo}_{2}$ at $85-90 \%$ Nebulised salbutamol $5 \mathrm{mg}$ 4-6 hourly

Nebulised ipratropium bromide $500 \mu \mathrm{g} 6$ hourly

Prednisolone $30 \mathrm{mg}$ once a day for a minimum of 5 days

Antibiotic agent

Non-invasive ventilation (118 patients)*

Bilevel positive pressure ventilation through a face or nasal mask

Inspiratory pressure initially $10 \mathrm{~cm} \mathrm{H}_{2} \mathrm{O}$, increased to $20 \mathrm{~cm} \mathrm{H}_{2} \mathrm{O}$

Expiratory pressure $5 \mathrm{~cm} \mathrm{H}_{2} \mathrm{O}$

Target duration first day 24 hours, second day 16 hours, third day 8 hours, fourth day discontinued Oxygen in the circuit to maintain $\mathrm{Spo}_{2}$ at $85-90 \%$ *Non-invasive ventilation was given in addition to standard treatment. count rate of 5\%. ${ }^{8}$ Masks, tubing, connectors, and headgear were replaced after 10 patients. We obtained the costs of cleaning and annual servicing from one centre and applied these to all. We recorded and valued all training, assuming that $50 \%$ of the training was given by a specialist registrar in the middle of the incremental pay scale and $50 \%$ by a $\mathrm{F}$ grade nurse specialist.

Costs of intensive care units-Each hospital provided the cost of a bed day in intensive care. We apportioned the cost in relation to length of stay, rounding up stays of less than one day to one day.

\section{Statistical analysis}

Results are given as means (standard deviations) for normally distributed data and as medians with ranges for non-normally distributed variables. All tests and P values are two tailed and were analysed on an intention to treat basis. We used $t$ tests to compare the group means and the Mann-Whitney $\mathrm{U}$ test to compare the medians. We applied Bonferroni's correction to multiple comparisons. We used Fisher's exact test to analyse two by two tables. We generated Kaplan-Meier curves for time data and used the log rank test to compare them. We used SPSS version 9 for our analyses. We applied non-parametric bootstrap techniques to the cost data for deaths avoided. We report the mean costs for 1000 bootstrap replications and assessed the significance of negative cost effectiveness ratios by using cost effectiveness acceptability curves.

\section{Results}

\section{Clinical outcomes}

One hundred and eighteen patients were randomised to non-invasive ventilation and 118 to standard treatment. The two groups had similar characteristics on admission. ${ }^{5}$ Of the group receiving standard treatment, 32/118 (27\% (SD 8\%)) met the primary clinical end point, "need for intubation," compared with 18/118 (15\% (SD 6.5\%)) in the group receiving non-invasive ventilation $(\mathrm{P}<0.02)$.

Of the 32 patients receiving standard treatment who met the failure criteria, only $75 \%$ (24) received ventilatory support either invasively or non-invasively (non-invasive ventilation alone $38 \%$ (12), non-invasive ventilation followed by invasive mechanical ventilation 9\% (3), invasive mechanical ventilation alone 28\% (9)). Eighteen patients in the non-invasive ventilation group met the failure criteria, of whom 7 (39\%) received invasive mechanical ventilation. Of the standard group 24/118 (20\% (SD 7.3\%)) died, compared with 12/118 $(10 \%$ (SD 5.5\%) in the non-invasive ventilation group $(\mathrm{P}=0.046){ }^{5}$ We found no statistical difference in need for intubation or mortality between the centres. Median length of stay in hospital was similar between the two groups, at 10 days (range: standard group 2-119 days, non-invasive ventilation group 4-137 days, $\mathrm{P}=0.27)$.

\section{Cost data}

Ward costs-Twenty five wards in 14 hospitals participated in this study. The ratios of nurses to patients ranged from 1:2.6 to $1: 13$, with a median of 1:11. Nine out of 14 centres provided detailed ward and overhead costs, which accounted for $85 \%$ of the patients recruited. The median cost of a bed day was $£ 108$ (range $£ 77$ to $£ 214$ ). The median value was 
applied to centres not providing full financial data. Non-invasive ventilation was associated with a modest increase in nursing workload of 26 minutes in the first eight hours of the admission (table 1). No difference became apparent after the first eight hours. The cost of this additional workload was $£ 4.45$ per patient receiving non-invasive ventilation.

Costs of intensive care centres-Ten centres admitted patients to intensive care. The mean cost of a bed day in intensive care was $£ 1228$ (95\% confidence interval $£ 1052$ to $£ 1404, \mathrm{n}=8$ ). The median length of stay in intensive care was similar between the standard group ( 5 days, range 1 to 53 days) and the non-invasive ventilation group ( 6 days, range 2 to 15 days, $\mathrm{P}=0.38$ ). In intensive care, the standard group $(n=12)$ consumed 116 bed days and the non-invasive ventilation group $(\mathrm{n}=7) 43$ bed days.

Costs of non-invasive ventilation-Table 2 shows the equipment purchased to set up the service. Allowing a three year lifespan for the ventilator and a two year lifespan for the consumables generates an equivalent annual cost of $£ 839$ and $£ 266$, respectively, at a discount rate of $5 \%$. The annual servicing cost was $£ 26$ per ventilator.

Table 2 Cost of purchasing equipment for each centre

\begin{tabular}{|c|c|c|}
\hline Equipment & No & Cost $(£)$ \\
\hline \multicolumn{3}{|l|}{ Masks: } \\
\hline Full face aircraft mask ${ }^{*}$ & 2 & 160.00 \\
\hline Small face mask $\dagger$ & 1 & 75.00 \\
\hline Nasal mask frame & 1 & 17.00 \\
\hline Small nasal mask & 1 & 36.00 \\
\hline Medium nasal mask & 1 & 36.00 \\
\hline Mouthpiece & 1 & 0.75 \\
\hline Nose clips & 1 & 1.00 \\
\hline \multicolumn{3}{|l|}{ Headgear: } \\
\hline Child soft cap & 1 & 28.00 \\
\hline Medium soft cap & 1 & 30.00 \\
\hline Large rescap & 1 & 21.00 \\
\hline Chin strap & 1 & 12.00 \\
\hline \multicolumn{3}{|l|}{ Connectors: } \\
\hline Elbow connectors & 2 & 1.80 \\
\hline Hooks for aircraft mask & 2 sets & 1.00 \\
\hline Whisper swivel exhale valve & 2 & 46.00 \\
\hline Oxygen port caps & 2 sets & 1.00 \\
\hline $22 \mathrm{~mm}$ tubing & 2 & 32.00 \\
\hline Masks, headgear, and connectors & Total & 519.55 \\
\hline \multicolumn{3}{|l|}{ Ventilator: } \\
\hline VPAPII ventilator & 1 & 2400.00 \\
\hline
\end{tabular}

*Friday Medical, London, UK.

†Respironics, Murrysville, USA

‡Resmed (UK) Limited, Abingdon, UK.

The mean amount of formal training given in the first three months of opening a ward was 7.6 (SD 3.6) hours. Thereafter each centre received 0.9 (SD 0.82) hours per month. The cost of providing training was £11-67 per hour.

The initial costs for equipment and the need for training are fixed costs that are independent of the number of patients treated in a centre. Additional costs were incurred by treating an individual patient. This included cleaning of equipment and replacement of masks and connectors after 10 patients (£11-75 per patient).

Where patients in the standard group were given non-invasive ventilation after standard treatment had
Table 1 Minutes of direct nursing care per patient per time period. Values are medians (ranges)

\begin{tabular}{lcccc} 
& \multicolumn{4}{c}{ Time period from randomisation } \\
\cline { 2 - 5 } Type of care & $\mathbf{0 - 1}$ hours $^{*}$ & $\mathbf{1 - 8}$ hours* & $\mathbf{8 - 2 4}$ hours & $\mathbf{2 4 - 4 8}$ hours \\
\hline Standard & $25(5-84)$ & $54(11-130)$ & $84(21-262)$ & $106(34-385)$ \\
\hline Non-invasive ventilation & $35(7-95)$ & $70(19-179)$ & $103(32-228)$ & $127(30-251)$ \\
\hline
\end{tabular}

${ }^{*} \mathrm{P}<0.05$.

failed, we assumed this treatment to have cost a 118th of the total study costs of non-invasive ventilation (equipment, training, and additional nursing time).

\section{Cost effectiveness}

Table 3 shows the total hospital costs in relation to in-hospital mortality, the primary outcome for the health economic analysis. Non-invasive ventilation was associated with a $£ 49362$ reduction in costs and a $50 \%$ reduction in mortality, with an additional 12 patients being discharged.

The main area of cost saving was in the use of intensive care units. The cost per patient in each group was skewed because of the high cost of patients admitted to intensive care. We therefore applied nonparametric bootstrapping to the cost data for deaths avoided. We performed 1000 bootstrap replications, and the mean costs were $£ 2800$ (95\% confidence interval $£ 1896$ to $£ 4388$ ) for the group receiving standard treatment and £2155 (£1742 to £2966) for the non-invasive ventilation group. The mean cost difference between the treatments shows a saving of $£ 645$ per patient receiving non-invasive ventilation ( $£ 2310$ to £386). The results indicate that noninvasive ventilation is a dominant strategy (more effective and less costly). However, the magnitude of negative incremental cost effectiveness ratios is not informative, and several problems are associated with such confidence intervals. We generated a cost effectiveness acceptability curve, which is used to incorporate the uncertainty around the estimates of mean costs and outcomes and the maximum (or ceiling) incremental cost effectiveness ratio that the decision maker would consider acceptable. The curve showed an $80 \%$ probability that non-invasive ventilation has a negative cost effectiveness ratio, meaning that it is cheaper and more effective. At a ceiling cost of $£ 5000$ per death prevented, the probability is $95 \%$ that non-invasive ventilation is more cost effective than standard treatment.

Table 3 Cost effectiveness of ward based non-invasive ventilation in reducing mortality in hospital in two groups of patients $(n=236)$

\begin{tabular}{|c|c|c|}
\hline & $\begin{array}{l}\text { Standard treatment } \\
\quad(n=118)\end{array}$ & $\begin{array}{l}\text { Non-invasive ventilation } \\
\qquad(\mathrm{n}=118)\end{array}$ \\
\hline \multicolumn{3}{|l|}{ Costs $(\mathfrak{£})$ : } \\
\hline Ward & 127355 & 139243 \\
\hline Non-invasive ventilation & $3390^{*}$ & 26664 \\
\hline Additional non-invasive ventilation nursing & $67^{*}$ & 525 \\
\hline Intensive care unit & 142576 & 52981 \\
\hline Total & 337435 & 288073 \\
\hline \multicolumn{3}{|l|}{ Effectiveness of intervention: } \\
\hline No of deaths & 24 & 12 \\
\hline No discharged & 98 & 108 \\
\hline Saving with non-invasive ventilation $(£)$ & - & 49362 \\
\hline Deaths avoided with non-invasive ventilation & - & 12 \\
\hline
\end{tabular}

${ }^{*}$ Cost due to the use of non-invasive ventilation after meeting failure criteria. 


\section{Modelling}

Because most costs for non-invasive ventilation are related to fixed costs the analyses may be sensitive to the number of patients treated in a centre. Hence we modelled the costs and effects of providing and not providing non-invasive ventilation in a typical hospital in the United Kingdom (population 250 000, standardised death rate for chronic obstructive pulmonary disease 100). A typical hospital will admit 72 patients per year with respiratory acidosis $\left(\mathrm{pH}<7.35\right.$ and $\mathrm{PaCO}_{2}<6$ $\mathrm{kPa}$ ) after immediate management. ${ }^{9}$ Fifty six will have a $\mathrm{pH}$ between 7.25 and 7.35. When a three day duration of non-invasive ventilation is assumed two ventilators will meet the demand for these 56 patients on $99 \%$ of days (calculated by using Poisson distribution). ${ }^{10}$

The box shows a comparison of the annual costs and effects of providing a non-invasive ventilation service for these 56 patients in a typical UK hospital compared with a standard service. We modelled two intubation rates in the standard group. In the randomised controlled trial 75\% (24) of patients failing in the standard group received ventilatory support; $38 \%$ (12) received non-invasive ventilation alone; and $38 \%$ (12) received invasive mechanical ventilation before or after non-invasive ventilation. Where no non-invasive ventilation service exists only invasive mechanical ventilation will be available; the expected rate of invasive mechanical ventilation in a UK hospital without non-invasive ventilation could therefore fluctuate between $38 \%$ and $75 \%$. The provision of a non-invasive ventilation service generates a saving of $£ 12351$ per year in the setting of low rates of intubation and $£ 53078$ in the setting with higher rates of intubation. This is achieved through preventing three and nine admissions to intensive care, respectively. From the hospital's perspective the provision of non-invasive ventilation would incur costs only if the use of intensive care units fell by $55 \%$.

\section{Discussion}

The health economic analysis of the randomised controlled trial showed that non-invasive ventilation is a dominant strategy from the hospital's perspective for patients with chronic obstructive pulmonary disease who have mild to moderate acidosis. The procedure reduced overall costs and reduced in-hospital mortality. Dominant strategies generate negative cost effectiveness ratios, which can be difficult to interpret. For example, if an intervention generates a $£ 1000$ saving for 10 lives (£100 saving per life saved), doubling the effectiveness generates a figure of a $£ 50$ saving per life saved. This seems financially less attractive but in reality is a superior outcome. We therefore generated a cost effectiveness acceptability curve to overcome the problems of a negative cost effectiveness ratio and the ratio's 95\% confidence interval. This indicated an 80\% probability that the non-invasive ventilation will generate a saving per life saved and a $95 \%$ probability that each life saved will cost less than $£ 5000$.

A notable proprtion of the costs of non-invasive ventilation are fixed costs are and therefore influenced by throughput. We therefore modelled the analysis to the annual needs of a typical hospital in the United Kingdom. When we used this different method of analysis and the $95 \%$ confidence interval for clinical outcomes rather than the costs, non-invasive ventilation was still associated with savings and a superior clinical outcome. From the hospital's perspective the provision of non-invasive ventilation would incur costs only if the use of intensive care units fell by $55 \%$. Internationally, intubation rates in the United Kingdom are considered low, and a further reduction to such low levels is clinically unrealistic and would almost certainly be associated with a higher mortality. Moreover such a change in practice is unlikely because of international standardisation and the development of international and global guidelines. Although these analyses show a saving to the hospital as a whole, costs are increased on the respiratory wards and this should be considered by clinicians and managers who are setting up non-invasive ventilation services. However, this cost was more than offset by the savings in costs of intensive care units.

\section{Cost effectiveness}

The bootstrapping analyses and the modelling indicate that our conclusions are robust and that non-invasive ventilation is a highly cost effective intervention. We are unaware of any similar prospective cost effectiveness analysis of non-invasive ventilation in the ward setting. However, in the intensive care setting non-invasive

\section{Costs and effects of providing a non-invasive ventilation service in a typical UK hospital treating 56 patients per year}

Non-invasive ventilation service

Ventilator and consumables: £2262

Replacement masks: $£ 658$

Training: £139

Additional nursing: £249

Annual cost: $£ 3308$

Patients admitted to intensive care unit for invasive mechanical ventilation:

No (95\% CI): 3 (2 to 5)

Cost (95\% CI) of intensive care unit: £21 959

(£12 671 to $£ 31246$ )

Total cost per year with non-invasive ventilation service: £25 267

\section{Standard service}

Low rate $(38 \%)$ of invasive mechanical ventilation in intensive care unit:

No of patients admitted: 6

Cost of intensive care unit: $£ 37618$

High rate $(75 \%)$ of invasive mechanical ventilation in intensive care unit:

No of patients admitted: 12

Cost of intensive care unit: $£ 78345$

Cost saving achieved by non-invasive mechanical ventilation

Low rate (38\%): $£ 12351$ (plus 3 admissions to intensive care unit)

High rate (75\%): $£ 53078$ (plus 9 admissions to intensive care)

Note: Category "Ventilator and consumables" assumes three year lifespan for ventilators and a two year lifespan for initial consumables. Category "Replacement masks" assumes that masks, headgear, and connectors were replaced after 10 patients. Category "Cost of intensive care unit" applies the median cost of an admission to intensive care (£6610). 
ventilation has been shown consistently to reduce the need for intubation by between $52 \%$ and $87 \%,{ }^{1-4}$ and in the largest study of intensive care units it has also been shown to reduce mortality by $65 \% .^{1}$ By using predefined criteria our randomised controlled trial shows a $44 \%$ reduction in the need for intubation and a $50 \%$ reduction in in-hospital mortality, ${ }^{5}$ which confirms the assumption of Bott et al that ward based non-invasive ventilation can reduce mortality. ${ }^{11}$ In view of this level of effectiveness and the large difference in cost between care on a ward and in an intensive care unit, it is not surprising that ward based non-invasive ventilation has been found to be highly cost effective. Keenan et al have conducted an economic evaluation of non-invasive ventilation for severe acute exacerbations of chronic obstructive pulmonary disease on the basis of a theoretical model. ${ }^{12}$ This model involved decision tree analysis constructed from a meta-analysis of published randomised controlled trials. They too concluded that non-invasive ventilation was a dominant strategy for severe exacerbations of chronic obstructive pulmonary disease.

\section{International perspective}

Our study must, however, be put into an international perspective. In many European countries and in North America non-invasive ventilation would not be considered an appropriate treatment on the ward. In the United Kingdom, however, intensive care beds are in short supply, and if patients with chronic obstructive pulmonary disease are to be offered non-invasive ventilation this must usually happen on the ward. Availability of intensive care beds also explains the low intubation rates and higher mortality found in this and other studies from the United Kingdom. ${ }^{11}$ The features of the UK setting may reduce both the generalisability of the mortality data and the cost effectiveness analysis to countries with better provision of intensive care units. However, for the United Kingdom, non-invasive ventilation for patients with mild to moderate acidosis due to decompensated chronic obstructive pulmonary disease is a highly effective technique that improves clinical outcomes, reduces demand for intensive care, and, from the hospital's perspective, reduces costs.

We thank Professor Christine Godfrey, Centre for Health Economics at the University of York, for advice on methods; Amanda Farrin and Vicky Allgar for statistical advice; ResMed (UK) for the loan of the ventilators; and the consultants, junior doctors, nursing staff, and physiotherapists at all 14 centres for help in conducting the trial.

Contributors: PKP and MWE were responsible for the study design, analysis, and writing of the paper. PKP and JLO were responsible for the education of staff and conduct of the trial. PKP was responsible for the statistical analysis and PKP and SP for the health economic analysis. PKP is guarantor for the study. Funding: Northern and Yorkshire NHS Executive. Ventilators loaned by ResMed (UK).

\section{What is already known on this topic}

Non-invasive ventilation reduces the need for intubation and mortality in hospital in patients with acute exacerbations of chronic obstructive pulmonary disease and acute respiratory failure

The procedure is feasible in the ward or intensive care environment

\section{What this study adds}

Non-invasive ventilation given on wards reduces the need for intubation by $44 \%$ and halves mortality in hospital in patients with chronic obstructive pulmonary disease and mild to moderate acidosis

The early use of non-invasive ventilation on the ward reduces costs and improves outcomes compared with traditional medical treatment

The main cost saving is in preventing the use of intensive care facilities

Competing interests: MWE receives research funding from ResMed (UK).

Ethical approval: The study was approved by the multicentre research ethics committee South West Thames and the local research ethics committees from the centres.

1 Brochard L, Mancebo J, Wysocki M, Lofaso F, Conti G, Rauss A, et al. Non-invasive ventilation for acute exacerbations of chronic obstructive pulmonary disease. $N$ Engl J Med 1995;333:817-22.

2 Kramer N, Meyer TJ, Meharg J, Cece RD, Hill NS. Randomised, prospective trial of noninvasive positive pressure ventilation in acute respiratory failure. Am J Respir Crit Care Med 1995;151:1799-806.

3 Martin TJ, Hovis JD, Costantino JP, Bierman MI, Donahue MP, Rogers RM, et al. A randomised prospective evaluation of non-invasive $\mathrm{RM}$, et al. A randomised prospective evaluation of non-invasive
ventilation for acute respiratory failure. Am J Respir Crit Care Med ventilation for acute respiratory failure. Am J Respir Crit Care Med 2000;161:807-13.

4 Celikel T, Sungur M, Ceyhan B, Karakurt S. Comparison of non-invasive positive pressure ventilation with standard medical therapy in hypercapnic acute respiratory failure. Chest 1998;114:1636-42.

5 Plant PK, Owen JL, Elliott MW. Early use of non-invasive ventilation for acute exacerbations of chronic obstructive pulmonary disease on general respiratory wards: a multicentre randomised controlled trial. Lancet 2000;355:1931-5

6 Kollef MH. Non-invasive ventilation for chronic obstructive pulmonary disease. Lancet 2000; 356:956-7.

7 Drummond MF, Stoddart GL, Torrance GW. Cost-effectiveness analysis. In: Methods for the economic evaluation of health care programmes. 1st ed. In: Methods for the economic evaluation of health
Oxford: Oxford University Press, 1987:74-111.

8 Oxford: Oxford University Press, 1987:74-111. the economic evaluation of health care programmes. 1st ed. Oxford: Oxford University Press, 1987:39-74.

9 Plant PK, Owen JL, Elliott MW. One-year period prevalence study of respiratory acidosis in acute exacerbations of COPD; implications for the provision of non-invasive ventilation and oxygen administration. Thorax 2000; 55:550-4

10 Altman DG. Theoretical distributions. In: Practical statistics for medical research. London: Chapman and Hall, 1991:48-73.

11 Bott J, Carroll MP, Conway JH, Keilty SEJ, Ward EM, Brown AM, et al. Randomised controlled trial of nasal ventilation in acute ventilatory failure due to chronic obstructive airways disease. Lancet 1993;341:1555-7.

12 Keenan SP, Gregor J, Sibbald WJ, Cook D, Gafni A. Noninvasive positive pressure ventilation in the setting of acute exacerbations of chronic obstructive pulmonary disease: more effective and less expensive. Crit Care Med 2000;28:2094-102.

(Accepted 6 March 2003) 